\title{
Simulation of the $\mathrm{A}-\mathrm{X}$ and $\mathrm{B}-\mathrm{X}$ transition emission spectra of the $\mathrm{InCl}$ molecule in low pressure plasmas
}

\author{
S. Briefia,b,*, U. Fantza,b \\ ${ }^{a}$ Lehrstuhl für Experimentelle Plasmaphysik, Universität Augsburg, Universitätsstr. 1, \\ 86135 Augsburg, Germany \\ ${ }^{b}$ Max-Planck-Institut für Plasmaphysik, EURATOM Association, Boltzmannstr. 2, 85748 \\ Garching, Germany
}

\begin{abstract}
Low pressure plasmas containing indium halides as radiators are discussed for lighting applications as efficient alternative to mercury-containing fluorescent lamps. To gain insight in plasma parameters like the vibrational and rotational temperature of the molecule, the near UV emission spectra of the indium halides arising from the $\mathrm{A}^{3} \Pi_{0^{+}} \rightarrow \mathrm{X}^{1} \Sigma^{+}$and the $\mathrm{B}^{3} \Pi_{1} \rightarrow \mathrm{X}^{1} \Sigma^{+}$transitions are simulated. Such a simulation requires Franck-Condon factors and vibrationally resolved transition probabilities which are not available in the literature for $\mathrm{InCl}$. Therefore, they have been calculated by solving the Schrödinger equation using the Born-Oppenheimer approximation. The values of the Franck-Condon factors and the transition probabilities are presented. For the A $-\mathrm{X}$ transition a good match of the simulated and measured spectra could be achieved but for the $\mathrm{B}-\mathrm{X}$ transition neither the relative intensity nor the wavelength could be reproduced. This indicates that for the $\mathrm{B}$ state the values of the molecular constants, the potential curve and/or the electronic dipole transition moment of the $\mathrm{B}-\mathrm{X}$ transition are inaccurate. Despite this mismatch the rotational and vibrational temperatures of the molecule can still be determined using the A - X transition.
\end{abstract}

Keywords: Indium chloride, Molecular emission, Franck-Condon factors, Transition probabilities

\section{Introduction}

The utilization of indium halides as radiator in low pressure discharges is discussed as efficient alternative to common fluorescent lamps which contain hazardous mercury [1]. The desired emission is located in the near UV spectral range between 300 and $400 \mathrm{~nm}$ which allows for a small Stokes shift (the energy lost in the conversion process of UV into visible light by means of a phosphor).

\footnotetext{
* Corresponding author

Email address: stefan.briefi@physik.uni-augsburg.de (S. Briefi)
} 
The emission arises from both the $\mathrm{A}^{3} \Pi_{0^{+}} \rightarrow \mathrm{X}{ }^{1} \Sigma^{+}$and the $\mathrm{B}^{3} \Pi_{1} \rightarrow \mathrm{X}{ }^{1} \Sigma^{+}$ transition (an exemplary emission spectrum of $\mathrm{InCl}$ is shown in figure 1). Investigations of $\mathrm{InBr}, \mathrm{InCl}$ and $\mathrm{InI}$ already proved the high efficiency of such a kind of discharge lamp [2]. However, in order to maximize the near UV radiation systematically, the population mechanisms of the molecular states involved in the desired transitions have to be investigated. This can be achieved by simulating the relative emission spectra and fitting it to the measured spectrum [3]. Such a simulation calculates the vibrational and rotational population based on molecular constants, Franck-Condon factors and vibrationally resolved transition probabilities. For $\mathrm{InBr}$ such a simulation has already been realized successfully [4].

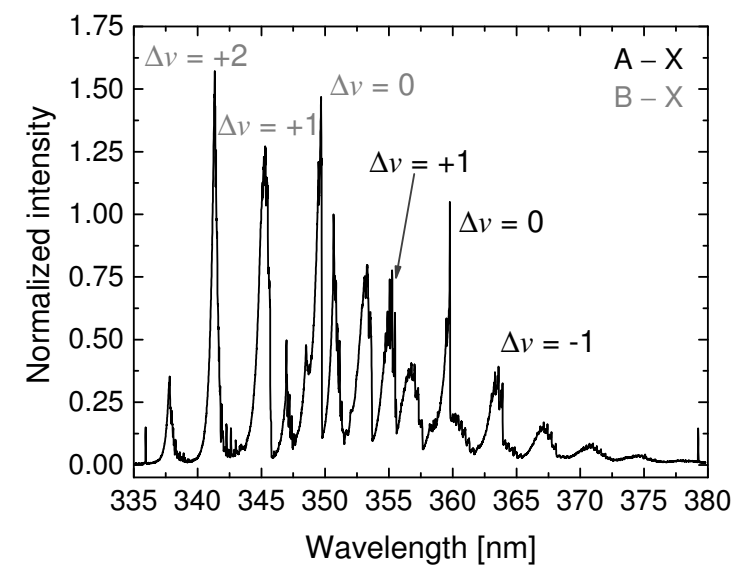

Figure 1: Exemplary near UV emission spectrum of $\mathrm{InCl}$ arising from the $\mathrm{A}-\mathrm{X}$ and the $\mathrm{B}-\mathrm{X}$ transition. Some exemplary sequences $\Delta v=v^{\prime}-v^{\prime \prime}$ of the electronic transitions are labelled.

In this paper the simulation of the emission spectrum is carried out for the $\mathrm{InCl}$ molecule. In general it is based on the same computing method as for $\mathrm{InBr}$, but the molecule-specific parameters such as molecular constants, FranckCondon factors and transition probabilities are substituted. For InCl neither Franck-Condon factors nor vibrationally resolved transition probabilities can be found in the literature. Therefore this data was calculated from potential curves and electronic dipole transition moments using the program TraDiMo [5]. The input parameters and the results of this calculation are described in section 2 . The comparison of simulated and measured spectra is carried out in section 3 .

\section{Simulation of the emission spectrum}

Only a brief description of the calculation method is given here, the reader is referred to [4] for a more detailed explanation. The simulation of the $\mathrm{InCl}$ emission is based on the calculation of the vibrational and rotational populations 
of the electronic ground state $\mathrm{X}$ and the first two excited electronic states $\mathrm{A}$ and $\mathrm{B}$ assuming Boltzmann distributions with the vibrational temperature $T_{V i b}$ and the rotational temperature $T_{R o t}$. The assumption of such a distribution is reasonable as the energy difference of the single vibrational and rotational states $\left(\Delta E_{V i b} \approx 0.03 \mathrm{eV}, \Delta E_{R o t} \approx 3 \times 10^{-5} \mathrm{eV}\right)$ is low compared to the gas temperature (typically above $800 \mathrm{~K}$ ) or the electron temperature (typically just below $1 \mathrm{eV}$ ). The first 25 vibrational states and the first 300 rotational states of each electronic state are included in the calculations as this proved to be necessary to achieve a good match between simulation and measurement for the InBr molecule [4].

The vibrational population is calculated in the electronic ground state and transferred to the A or B state by applying Franck-Condon factors. This corresponds to a population of the excited states by electron impact excitation out of the ground state. As the redistribution of the rotational population in the excited electronic state via collisions with the background gas happens on a much shorter time scale than the radiative decay [6], the rotational population can be calculated directly in the excited electronic states. The relative intensity of the emission is then derived from the calculated population in the excited rovibronic state, the vibrationally resolved transition probabilities, the Hönl-London factors and constants of the particular transition such as statistical weights and the frequency of the emission line. For each emission line a Gaussian line profile having the full width at half maximum of the apparatus profile is calculated and folded with the relative intensity of the line. By adding up all emission lines from both the $\mathrm{A}-\mathrm{X}$ and the $\mathrm{B}-\mathrm{X}$ transition, the simulated spectrum is obtained.

As mentioned above, the simulation requires molecular constants, FranckCondon factors and vibrationally resolved transition probabilities as input parameters. As these parameters are isotope-specific, the isotopes of $\mathrm{InCl}$ having a relevant natural abundance must be considered in the simulation. The two isotopes ${ }^{115} \mathrm{In}^{35} \mathrm{Cl}$ and ${ }^{115} \mathrm{In}^{37} \mathrm{Cl}$ (natural abundance $72.51 \%$ and $23.20 \%$ resp.) are taken into account whereas the isotopes ${ }^{113} \mathrm{In}^{35} \mathrm{Cl}$ and ${ }^{113} \mathrm{In}^{37} \mathrm{Cl}(3.25 \%$ and $1.04 \%$ resp.) are neglected due to their low abundance.

The molecular constants of $\mathrm{InCl}$ are taken from [7] which is a recent review of the spectroscopic constants of the diatomic indium halide molecules. However, Franck-Condon factors $(F C F)$ and vibrationally resolved transition probabilities $A_{i k}^{v^{\prime} v^{\prime \prime}}$ ( $i$ denotes the upper electronic state, $v^{\prime}$ the upper vibrational state, $k$ and $v^{\prime \prime}$ the corresponding lower states) are not available in the literature for InCl. Therefore they have been calculated using the program TraDiMo which derives the eigenvalues and vibrational wave functions via numerically solving the Schrödinger equation (for a detailed description of the program see [5]). The calculations require Born-Oppenheimer potential curves, electronic dipole transition moments and the reduced mass of the molecule as input parameters. The overlap integral of two vibrational wave functions in different electronic states yields the $F C F$ whereas the $A_{i k}^{v^{\prime} v^{\prime \prime}}$ are calculated from the overlap of the vibrational wave functions with the electronic dipole transition moments.

Four different sets of potential curves for the relevant states of $\mathrm{InCl}$ are avail- 
able: RKR potential curves [7], potential curves from two different quantum mechanical calculations $[8,9]$ and those obtained by a pseudopotential approximation [10]. As the data for RKR potential curves are only available up to a vibrational quantum number of 16 they are fitted using Morse potential curves which can be derived using molecular constants taken from [7]. The electronic dipole transition moments are given by [8] or by [11]. TraDiMo calculations were carried out with each possible set of potential curves and dipole transition moments for both relevant isotopes of $\mathrm{InCl}$.

As the different potential curves and dipole transition moments deviate from each other, the obtained sets of $F C F$ and $A_{i k}^{v^{\prime} v^{\prime \prime}}$ and therefore also the simulated spectra show deviations. Simulations were carried out with all sets of obtained $F C F$ and $A_{i k}^{v^{\prime} v^{\prime \prime}}$ and compared to measured emission spectra. For the $\mathrm{A}-\mathrm{X}$ transition a satisfying agreement of simulated and measured spectra could only be achieved with the data derived using the Morse potential curves and the electronic dipole transition moments of [11]. For the $\mathrm{B}-\mathrm{X}$ transition the relative intensity and the wavelengths of the emission lines could not be reproduced properly with any parameter set. The smallest deviation between simulation and measurement was achieved by using the $F C F$ and $A_{i k}^{v^{\prime} v^{\prime \prime}}$ obtained from the Morse potential curves and the electronic dipole transition moments of [11]. An exemplary comparison of simulation and measurement demonstrating the deviations is shown in section 3. Possible reasons of the mismatch of the B $\mathrm{X}$ transition are also discussed in this section.

As the calculation of Morse potential curves is based on molecular constants slightly different curves are obtained for each isotope which also lead to slightly different values of the determined $F C F$ and $A_{i k}^{v^{\prime} v^{\prime \prime}}$. Similar to the InBr simulation [4], TraDiMo calculations have been carried out on the one hand considering the mass of both relevant $\mathrm{InCl}$ isotopes and on the other hand with the averaged mass. The simulation results obtained by considering the isotopic composition for the Franck-Condon factors and the transition probabilities and by using the data obtained at the averaged mass only differs in the range of a few per cent. Therefore the $F C F$ and $A_{i k}^{v^{\prime} v^{\prime \prime}}$ obtained with the averaged mass are used in the simulation for simplicity (the corresponding Morse potential curves are shown in figure 2). The values are given in tables A.1 to A.8 in the Appendix. Considering the uncertainty of the obtained absolute values (a detailed discussion can be found in [4]), Franck-Condon factors that are smaller than $1 \times 10^{-5}$ and transition probabilities smaller than $1 \mathrm{~s}^{-1}$ have been replaced by zero.

\section{Comparison of simulated and measured spectra}

Figure 3 shows a sketch of the experimental setup used for the measurements of the emission spectra. Discharges are generated in sealed cylindrical vessels (diameter $2.5 \mathrm{~cm}$, length $18 \mathrm{~cm}$ ) via inductive RF coupling at a frequency of $13.56 \mathrm{MHz}$ and a power of $100 \mathrm{~W}$. The vessels are filled with argon at a pressure of one mbar and a few $\mathrm{mg}$ of $\mathrm{InCl}$ salt. As the vapour pressure of $\mathrm{InCl}$ is very low at ambient temperature, the discharge vessel is placed in a heat container made out of aerated autoclaved concrete and heated up to several hundred 


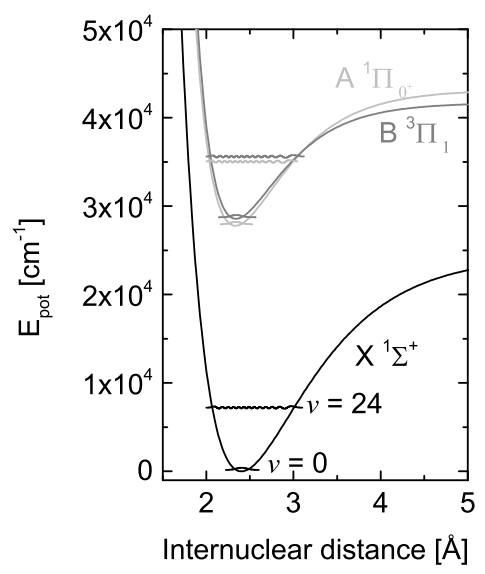

Figure 2: Morse potential curves of the $\mathrm{X}, \mathrm{A}$ and $\mathrm{B}$ states utilized for the calculation of the $F C F$ and $A_{i k}^{v^{\prime} v^{\prime \prime}}$. The vibrational wavefunctions of the states with $v=0$ and $v=24$ calculated with the program TraDiMo are also shown.

degrees with hot air. In order to control the evaporated amount of the indium halide, a setup has been realized where the cold spot location is well-defined and the cold spot temperature (which determines the indium halide density in gas phase) is adjustable. For details on the setup and its performance see [12]. The optical emission spectroscopy measurements are performed at an axial (radial centred) line-of-sight (LOS) utilizing a wavelength and intensity calibrated high resolution spectrometer equipped with a CCD detector (focal length $750 \mathrm{~mm}$, grating 1800 lines $/ \mathrm{mm}$ ). The full width at half maximum of the apparatus profile is $25 \mathrm{pm}$ at $370 \mathrm{~nm}$. Such a high spectral resolution is necessary in order to allow for a determination of the vibrational and the rotational temperature with high accuracy [4]. However, it should be noted that the single emission lines always overlap as the line width determined by natural, pressure and Doppler broadening is larger than the wavelength separation of the individual lines. Therefore, the high resolution is required to resolve the detailed structure of the whole band and not to resolve the single rotational branches.

Figure 4 shows an exemplary emission spectrum obtained from an $\mathrm{InCl}$ containing argon discharge at a cold spot temperature of $221{ }^{\circ} \mathrm{C}$ together with a simulation. The spectra are normalized to the maximum intensity of the $\Delta v=v^{\prime}-v^{\prime \prime}=+2$ sequence of the $\mathrm{A}-\mathrm{X}$ transition. It can be seen that the $\mathrm{A}$ - X transition (which is primarily located above $350 \mathrm{~nm}$ ) is reproduced quite well whereas neither the wavelength nor the relative intensity of the B - X transition (mainly located below $350 \mathrm{~nm}$ ) matches the measurement. Looking at the spectra in more detail the mismatch of the $\Delta v=+3,+2$ and +1 sequences of the $\mathrm{B}-\mathrm{X}$ transition is obvious (figure $5 \mathrm{a}$ )) whereas even the details of the $\Delta v=+1$ sequence of the $\mathrm{A}-\mathrm{X}$ transition are reproduced very well (see figure 


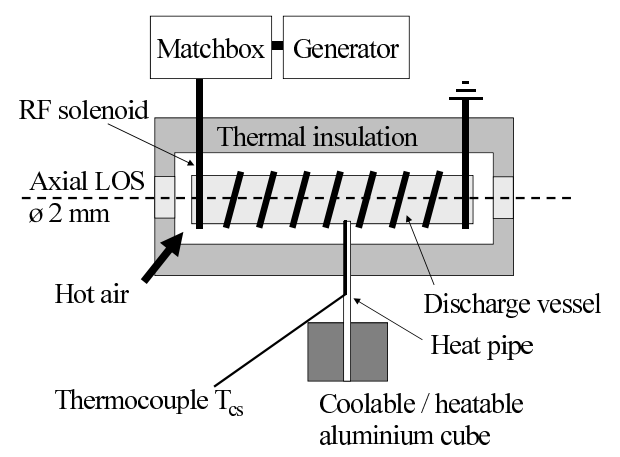

Figure 3: Sketch of the experimental setup.

$5 \mathrm{~b}))$.

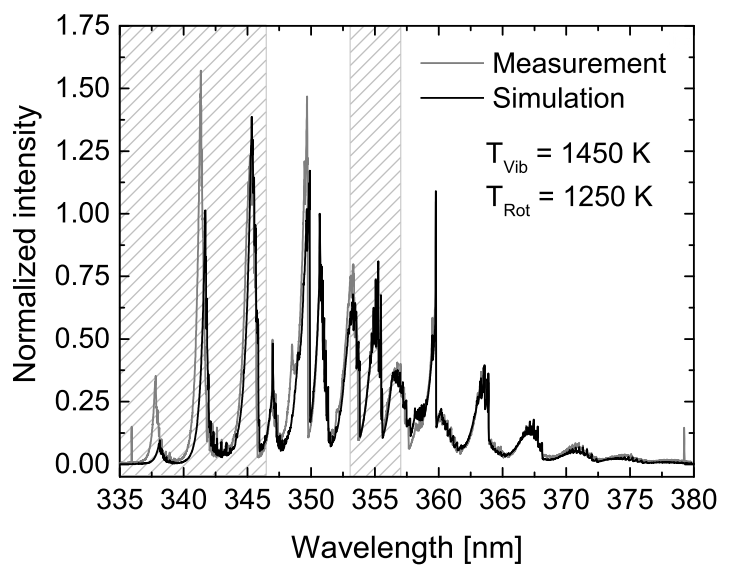

Figure 4: Measurement and simulation of an exemplary $\mathrm{InCl}$ emission spectrum. The parameters of the simulation are: $T_{V i b}=1450 \pm 50 \mathrm{~K}, T_{R o t}=1250 \pm 50 \mathrm{~K}$ (determined from the A $-\mathrm{X}$ transition). The two highlighted parts of the spectrum are shown in figure 5 in more detail.

In figure 6 the simulated $\Delta v=+1$ sequence of the $\mathrm{A}-\mathrm{X}$ transition is shown for a variation of the vibrational and of the rotational temperature. It can be seen that the vibrational temperature primarily determines the relative intensity of the whole sequence whereas the rotational temperature mainly influences the relative intensity in the left edge of the sequence. Due to the good reproduction of the measurement by the simulation concerning the $\mathrm{A}-\mathrm{X}$ transition and especially the $\Delta v=+1$ sequence, the vibrational and rotational temperatures can be determined with high accuracy. The error can be estimated to be $\Delta T_{V i b}=\Delta T_{R o t}= \pm 50 \mathrm{~K}$.

In order to discuss possible reasons for the deviation of the wavelength posi- 


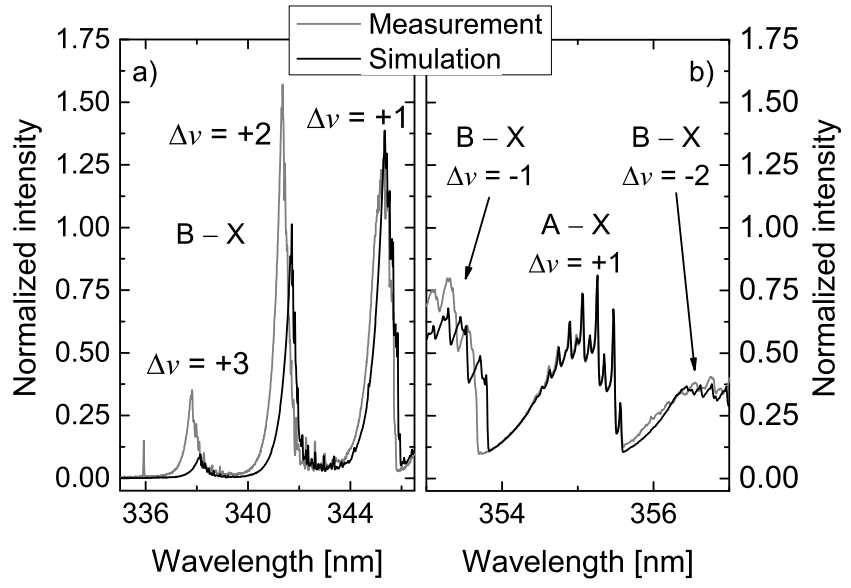

Figure 5: Details of the same spectrum as shown in figure 4. It can be seen that the simulation reproduces even the details of the $\mathrm{A}-\mathrm{X}$ transition very well in contrast to the $\mathrm{B}-\mathrm{X}$ transition.

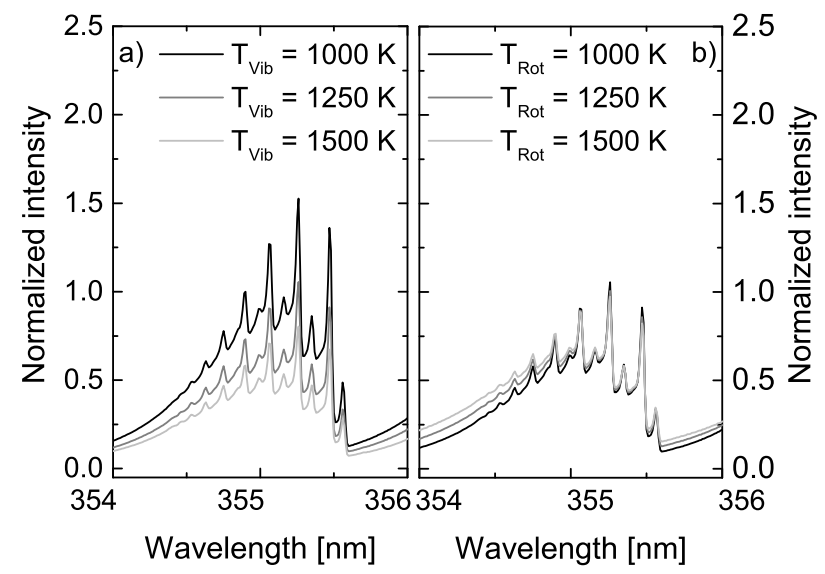

Figure 6: Simulation of the $\Delta v=+1$ sequence of the $\mathrm{A}-\mathrm{X}$ transition with different values of $T_{V i b}$ at a fixed rotational temperature of $1000 \mathrm{~K}$ (left part) and with different values of $T_{R o t}$ at a fixed vibrational temperature of $1000 \mathrm{~K}$ (right part). 
tion and of the relative intensity of the $\mathrm{B}-\mathrm{X}$ transition, the input parameters of the simulation which influence these quantities have to be considered. The wavelength of the particular emission lines is calculated using only molecular constants of the involved electronic states. Therefore, the mismatch between simulation and measurement regarding the wavelength position can be assigned to be caused by the molecular constants. As the wavelength position of the A $\mathrm{X}$ transition is reproduced well, it can be assumed that the molecular constants of the $\mathrm{X}$ state are correct and the deviation has to be caused by inaccurate molecular constants of the B state. The mismatch of the relative intensity of the simulated emission can be caused on the one hand by inaccurate potential curves and on the other hand by an inaccurate electronic dipole transition moment for the $\mathrm{B}-\mathrm{X}$ transition. Again, as the $\mathrm{A}-\mathrm{X}$ transition is reproduced well, the assumption can be made that the potential curve of the $\mathrm{X}$ state is accurate. This narrows the possible causes for the deviation down to inaccuracies of the potential curve of the B state or of the electronic dipole transition moment of the $\mathrm{B}-\mathrm{X}$ transition.

\section{Conclusion}

Indium halides are discussed for lighting purposes as efficient alternative to hazardous mercury containing fluorescent lamps. To optimize the efficiency, insight in the molecular population processes is highly desirable. In order to achieve this goal a simulation of the relative intensity of the emission spectrum can be carried out. This simulation requires Franck-Condon factors and vibrationally resolved transition probabilities which were not available in the literature for $\mathrm{InCl}$. Therefore this data has been calculated using the program TraDiMo and different sets of potential curves and electronic dipole transition moments as input parameters. The best match between measured spectra and simulated ones has been achieved for the data derived from Morse potential curves and electronic dipole transition moments taken from [11]. Using this input data which is presented in the tables in the appendix, the simulation can be fitted to the measurement for the $\mathrm{A}-\mathrm{X}$ transition very well (especially to the $\Delta v=+1$ sequence) whereas the $\mathrm{B}-\mathrm{X}$ transition shows deviations concerning the wavelength position and the relative intensity.

In summary, it has been demonstrated that the adjustment of the simulation to the measured $\mathrm{InCl}$ emission spectrum is suitable for determining the vibrational and rotational temperature of the $\mathrm{InCl}$ molecule if the $\mathrm{A}-\mathrm{X}$ transition is considered.

\section{References}

[1] Kitsinelis S, Zissis G, Fokitis E. A strategy towards the next generation of low pressure discharge lamps: lighting after mercury. Journal of Physics D Applied Physics 2009;42(4):045209 (pages 8). 
[2] Hayashi D, Hilbig R, Körber A, Schwan S, Scholl R, Boerger M, et al. Lowpressure indium-halide discharges for fluorescent illumination applications. Applied Physics Letters 2010;96(6):061503 (pages 2).

[3] Fantz U. Basics of plasma spectroscopy. Plasma Sources Science and Technology 2006;15(4):S137-47.

[4] Briefi S, Fantz U. Simulation of the A - X and B - X transition emission spectra of the $\mathrm{InBr}$ molecule for diagnostics in low-pressure plasmas. Journal of Physics D: Applied Physics 2011;44(15):155202 (pages 3).

[5] Fantz U, Wünderlich D. Franck Condon factors, transition probabilities, and radiative lifetimes for hydrogen molecules and their isotopomeres. Atomic Data and Nuclear Data Tables 2006;92:853-973.

[6] Mulders HCJ. Spectroscopic Investigation of Indium Bromide for Lighting Purposes; 2010. PhD thesis, Eindhoven University of Technology.

[7] Mishra SK, Yadav RKS, Singh VB, Rai SB. Spectroscopic Studies of Diatomic Indium Halides. Journal of Physical and Chemical Reference Data 2004;33:453-70.

[8] Banerjee A, Das KK. Low-lying electronic states and spectroscopic properties of $\mathrm{InCl}$ and $\mathrm{InCl}^{+}$. Journal of Molecular Structure: THEOCHEM 2008;851:134-46.

[9] Zou W, Lin M, Yang X, Zhang B. Ab initio calculations on the ground and low-lying excited states of InCl. Journal of Chemical Physics 2003;119:3721-8.

[10] Schwerdtfeger P, Fischer T, Dolg M, Igel-Mann G, Nicklass A, Stoll H, et al. The accuracy of the pseudopotential approximation. I. An analysis of the spectroscopic constants for the electronic ground states of $\mathrm{InCl}$ and $\mathrm{InCl}_{3}$ using various three valence electron pseudopotentials for indium. Journal of Chemical Physics 1995;102:2050-62.

[11] Körber A, Hayashi D. Absorption and emission spectra of gaseous indium monohalides. XXVIII Int Conf on Phenomena in Ionized Gases (Prague) $2007 ;: 1224-7$.

[12] Briefi S. Note: Implementation of a cold spot setup for controlled variation of vapor pressures and its application to an $\mathrm{InBr}$ containing discharge lamp. Review of Scientific Instruments 2013;84(2):026106 (pages 3).

\section{Appendix}


Table A.1: Franck-Condon factors for the A $-\mathrm{X}$ transition, part 1 (from $v^{\prime \prime}=0$ to $v^{\prime \prime}=12$ ).

\begin{tabular}{|c|c|c|c|c|c|c|c|c|c|c|c|c|c|}
\hline$v^{\prime}$ & $v^{\prime \prime}=0$ & $v^{\prime \prime}=1$ & $v^{\prime \prime}=2$ & $v^{\prime \prime}=3$ & $v^{\prime \prime}=4$ & $v^{\prime \prime}=5$ & $v^{\prime \prime}=6$ & $v^{\prime \prime}=7$ & $v^{\prime \prime}=8$ & $v^{\prime \prime}=9$ & $v^{\prime \prime}=10$ & $v^{\prime \prime}=11$ & $v^{\prime \prime}=12$ \\
\hline 0 & 0.54639 & 0.30992 & 0.10703 & 0.02869 & 0.00642 & 0.00128 & 0.00022 & 0.00003 & 0 & 0 & 0 & 0 & 0 \\
\hline 1 & 0.35605 & 0.09366 & 0.27488 & 0.17829 & 0.07003 & 0.02071 & 0.00509 & 0.00106 & 0.00020 & 0.00003 & 0 & 0 & 0 \\
\hline 2 & 0.08759 & 0.38637 & 0.00046 & 0.16742 & 0.19546 & 0.10707 & 0.04012 & 0.01188 & 0.00289 & 0.00061 & 0.00011 & 0.00002 & 0 \\
\hline 3 & 0.00954 & 0.18074 & 0.30983 & 0.02540 & 0.07806 & 0.17584 & 0.13101 & 0.06073 & 0.02117 & 0.00593 & 0.00141 & 0.00029 & 0.00005 \\
\hline 4 & 0.00039 & 0.02799 & 0.25288 & 0.21672 & 0.07204 & 0.02528 & 0.13943 & 0.14055 & 0.07906 & 0.03206 & 0.01017 & 0.00270 & 0.00060 \\
\hline 5 & 0 & 0.00137 & 0.05199 & 0.30058 & 0.13876 & 0.10794 & 0.00315 & 0.10044 & 0.13791 & 0.09326 & 0.04330 & 0.01542 & 0.00452 \\
\hline 6 & 0 & 0 & 0.00287 & 0.07825 & 0.32838 & 0.08273 & 0.12673 & 0.00075 & 0.06636 & 0.12711 & 0.10238 & 0.05396 & 0.02135 \\
\hline 7 & 0 & 0 & 0 & 0.00466 & 0.10435 & 0.34249 & 0.04616 & 0.13118 & 0.00865 & 0.04006 & 0.11185 & 0.10658 & 0.06332 \\
\hline 8 & 0 & 0 & 0 & 0 & 0.00643 & 0.12873 & 0.34855 & 0.02407 & 0.12605 & 0.02042 & 0.02171 & 0.09487 & 0.10680 \\
\hline 9 & 0 & 0 & 0 & 0.00001 & 0 & 0.00787 & 0.15050 & 0.35087 & 0.01170 & 0.11560 & 0.03230 & 0.01006 & 0.07834 \\
\hline 10 & 0 & 0 & 0 & 0 & 0.00003 & 0.00005 & 0.00871 & 0.16916 & 0.35245 & 0.00534 & 0.10286 & 0.04249 & 0.00355 \\
\hline 11 & 0 & 0 & 0 & 0 & 0 & 0.00006 & 0.00016 & 0.00879 & 0.18437 & 0.35537 & 0.00237 & 0.08974 & 0.05037 \\
\hline 12 & 0 & 0 & 0 & 0 & 0 & 0 & 0.00010 & 0.00043 & 0.00804 & 0.19584 & 0.36087 & 0.00115 & 0.07731 \\
\hline 13 & 0 & 0 & 0 & 0 & 0 & 0 & 0 & 0.00014 & 0.00090 & 0.00653 & 0.20328 & 0.36971 & 0.00078 \\
\hline 14 & 0 & 0 & 0 & 0 & 0 & 0 & 0 & 0 & 0.00017 & 0.00164 & 0.00450 & 0.20633 & 0.38214 \\
\hline 15 & 0 & 0 & 0 & 0 & 0 & 0 & 0 & 0 & 0 & 0.00019 & 0.00271 & 0.00234 & 0.20450 \\
\hline 16 & 0 & 0 & 0 & 0 & 0 & 0 & 0 & 0 & 0 & 0.00002 & 0.00018 & 0.00407 & 0.00061 \\
\hline 17 & 0 & 0 & 0 & 0 & 0 & 0 & 0 & 0 & 0 & 0 & 0.00004 & 0.00013 & 0.00566 \\
\hline 18 & 0 & 0 & 0 & 0 & 0 & 0 & 0 & 0 & 0 & 0 & 0 & 0.00008 & 0.00006 \\
\hline 19 & 0 & 0 & 0 & 0 & 0 & 0 & 0 & 0 & 0 & 0 & 0 & 0 & 0.00013 \\
\hline 20 & 0 & 0 & 0 & 0 & 0 & 0 & 0 & 0 & 0 & 0 & 0 & 0 & 0 \\
\hline 21 & 0 & 0 & 0 & 0 & 0 & 0 & 0 & 0 & 0 & 0 & 0 & 0 & 0 \\
\hline 22 & 0 & 0 & 0 & 0 & 0 & 0 & 0 & 0 & 0 & 0 & 0 & 0 & 0 \\
\hline 23 & 0 & 0 & 0 & 0 & 0 & 0 & 0 & 0 & 0 & 0 & 0 & 0 & 0 \\
\hline 24 & 0 & 0 & 0 & 0 & 0 & 0 & 0 & 0 & 0 & 0 & 0 & 0 & 0 \\
\hline
\end{tabular}


Table A.2: Franck-Condon factors for the A $-\mathrm{X}$ transition, part $2\left(\right.$ from $v^{\prime \prime}=13$ to $\left.v^{\prime \prime}=24\right)$.

\begin{tabular}{|c|c|c|c|c|c|c|c|c|c|c|c|c|}
\hline$v^{\prime}$ & $v^{\prime \prime}=13$ & $v^{\prime \prime}=14$ & $v^{\prime \prime}=15$ & $v^{\prime \prime}=16$ & $v^{\prime \prime}=17$ & $v^{\prime \prime}=18$ & $v^{\prime \prime}=19$ & $v^{\prime \prime}=20$ & $v^{\prime \prime}=21$ & $v^{\prime \prime}=22$ & $v^{\prime \prime}=23$ & $v^{\prime \prime}=24$ \\
\hline 0 & 0 & 0 & 0 & 0 & 0 & 0 & 0 & 0 & 0 & 0 & 0 & 0 \\
\hline 1 & 0 & 0 & 0 & 0 & 0 & 0 & 0 & 0 & 0 & 0 & 0 & 0 \\
\hline 2 & 0 & 0 & 0 & 0 & 0 & 0 & 0 & 0 & 0 & 0 & 0 & 0 \\
\hline 3 & 0 & 0 & 0 & 0 & 0 & 0 & 0 & 0 & 0 & 0 & 0 & 0 \\
\hline 4 & 0.00011 & 0.00002 & 0 & 0 & 0 & 0 & 0 & 0 & 0 & 0 & 0 & 0 \\
\hline 5 & 0.00110 & 0.00022 & 0.00004 & 0 & 0 & 0 & 0 & 0 & 0 & 0 & 0 & 0 \\
\hline 6 & 0.00685 & 0.00180 & 0.00040 & 0.00007 & 0 & 0 & 0 & 0 & 0 & 0 & 0 & 0 \\
\hline 7 & 0.02756 & 0.00963 & 0.00274 & 0.00064 & 0.00013 & 0.00002 & 0 & 0 & 0 & 0 & 0 & 0 \\
\hline 8 & 0.07079 & 0.03377 & 0.01271 & 0.00388 & 0.00098 & 0.00020 & 0.00003 & 0 & 0 & 0 & 0 & 0 \\
\hline 9 & 0.10377 & 0.07643 & 0.03955 & 0.01602 & 0.00521 & 0.00140 & 0.00031 & 0.00005 & 0 & 0 & 0 & 0 \\
\hline 10 & 0.06331 & 0.09864 & 0.08015 & 0.04476 & 0.01938 & 0.00669 & 0.00191 & 0.00044 & 0.00007 & 0 & 0 & 0 \\
\hline 11 & 0.00063 & 0.05033 & 0.09229 & 0.08209 & 0.04931 & 0.02263 & 0.00828 & 0.00249 & 0.00060 & 0.00011 & 0.00001 & 0 \\
\hline 12 & 0.05594 & 0.00001 & 0.03962 & 0.08524 & 0.08275 & 0.05296 & 0.02577 & 0.00989 & 0.00313 & 0.00079 & 0.00015 & 0.00002 \\
\hline 13 & 0.06599 & 0.05958 & 0.00070 & 0.03094 & 0.07825 & 0.08210 & 0.05595 & 0.02855 & 0.01155 & 0.00380 & 0.00100 & 0.00020 \\
\hline 14 & 0.00090 & 0.05594 & 0.06170 & 0.00201 & 0.02415 & 0.07146 & 0.08074 & 0.05806 & 0.03110 & 0.01310 & 0.00451 & 0.00123 \\
\hline 15 & 0.39810 & 0.00156 & 0.04707 & 0.06279 & 0.00345 & 0.01892 & 0.06522 & 0.07876 & 0.05951 & 0.03327 & 0.01457 & 0.00520 \\
\hline 16 & 0.19737 & 0.41711 & 0.00311 & 0.03919 & 0.06326 & 0.00473 & 0.01494 & 0.05964 & 0.07640 & 0.06036 & 0.03504 & 0.01592 \\
\hline 17 & 0.00001 & 0.18458 & 0.43831 & 0.00615 & 0.03211 & 0.06348 & 0.00568 & 0.01197 & 0.05473 & 0.07388 & 0.06064 & 0.03650 \\
\hline 18 & 0.00730 & 0.00134 & 0.16609 & 0.46031 & 0.01147 & 0.02564 & 0.06377 & 0.00619 & 0.00977 & 0.05057 & 0.07125 & 0.06056 \\
\hline 19 & 0 & 0.00874 & 0.00536 & 0.14224 & 0.48121 & 0.01993 & 0.01966 & 0.06441 & 0.00625 & 0.00815 & 0.04709 & 0.06865 \\
\hline 20 & 0.00019 & 0.00004 & 0.00965 & 0.01268 & 0.11409 & 0.49854 & 0.03239 & 0.01414 & 0.06566 & 0.00588 & 0.00696 & 0.04424 \\
\hline 21 & 0 & 0.00024 & 0.00028 & 0.00971 & 0.02354 & 0.08349 & 0.50935 & 0.04952 & 0.00915 & 0.06778 & 0.00511 & 0.00608 \\
\hline 22 & 0 & 0.00001 & 0.00027 & 0.00083 & 0.00871 & 0.03759 & 0.05317 & 0.51051 & 0.07157 & 0.00491 & 0.07103 & 0.00405 \\
\hline 23 & 0 & 0 & 0.00003 & 0.00025 & 0.00178 & 0.00669 & 0.05378 & 0.02661 & 0.49915 & 0.09813 & 0.00176 & 0.07571 \\
\hline 24 & 0 & 0 & 0 & 0.00008 & 0.00019 & 0.00315 & 0.00401 & 0.07032 & 0.00770 & 0.47314 & 0.12788 & 0.00013 \\
\hline
\end{tabular}


Table A.3: Franck-Condon factors for the B $-\mathrm{X}$ transition, part 1 (from $v^{\prime \prime}=0$ to $v^{\prime \prime}=12$ ).

\begin{tabular}{|c|c|c|c|c|c|c|c|c|c|c|c|c|c|}
\hline$v^{\prime}$ & $v^{\prime \prime}=0$ & $v^{\prime \prime}=1$ & $v^{\prime \prime}=2$ & $v^{\prime \prime}=3$ & $v^{\prime \prime}=4$ & $v^{\prime \prime}=5$ & $v^{\prime \prime}=6$ & $v^{\prime \prime}=7$ & $v^{\prime \prime}=8$ & $v^{\prime \prime}=9$ & $v^{\prime \prime}=10$ & $v^{\prime \prime}=11$ & $v^{\prime \prime}=12$ \\
\hline 0 & 0.60396 & 0.28705 & 0.08506 & 0.01955 & 0.00368 & 0.00061 & 0.00008 & 0 & 0 & 0 & 0 & 0 & 0 \\
\hline 1 & 0.32792 & 0.16249 & 0.29240 & 0.15241 & 0.04973 & 0.01218 & 0.00243 & 0.00039 & 0.00005 & 0 & 0 & 0 & 0 \\
\hline 2 & 0.06338 & 0.40087 & 0.02222 & 0.21882 & 0.18349 & 0.07997 & 0.02430 & 0.00571 & 0.00107 & 0.00016 & 0.00002 & 0 & 0 \\
\hline 3 & 0.00464 & 0.13624 & 0.37634 & 0.00043 & 0.14151 & 0.18578 & 0.10417 & 0.03793 & 0.01037 & 0.00218 & 0.00037 & 0.00004 & 0 \\
\hline 4 & 0.00007 & 0.01321 & 0.19997 & 0.32363 & 0.01743 & 0.08238 & 0.17132 & 0.12014 & 0.05138 & 0.01594 & 0.00375 & 0.00069 & 0.00009 \\
\hline 5 & 0 & 0.00019 & 0.02365 & 0.25078 & 0.27089 & 0.04097 & 0.04336 & 0.14944 & 0.12850 & 0.06324 & 0.02199 & 0.00569 & 0.00115 \\
\hline 6 & 0 & 0 & 0.00027 & 0.03402 & 0.29013 & 0.22791 & 0.05969 & 0.02017 & 0.12628 & 0.13067 & 0.07299 & 0.02793 & 0.00793 \\
\hline 7 & 0 & 0 & 0.00004 & 0.00025 & 0.04279 & 0.32080 & 0.19680 & 0.07103 & 0.00779 & 0.10494 & 0.12871 & 0.08024 & 0.03356 \\
\hline 8 & 0 & 0 & 0 & 0.00011 & 0.00013 & 0.04890 & 0.34540 & 0.17696 & 0.07551 & 0.00208 & 0.08686 & 0.12404 & 0.08529 \\
\hline 9 & 0 & 0 & 0 & 0 & 0.00024 & 0 & 0.05167 & 0.36567 & 0.16712 & 0.07452 & 0.00016 & 0.07231 & 0.11808 \\
\hline 10 & 0 & 0 & 0 & 0 & 0 & 0.00045 & 0.00010 & 0.05071 & 0.38237 & 0.16627 & 0.06944 & 0.00016 & 0.06115 \\
\hline 11 & 0 & 0 & 0 & 0 & 0 & 0 & 0.00073 & 0.00074 & 0.04600 & 0.39541 & 0.17375 & 0.06142 & 0.00102 \\
\hline 12 & 0 & 0 & 0 & 0 & 0 & 0 & 0 & 0.00103 & 0.00229 & 0.03792 & 0.40381 & 0.18930 & 0.05136 \\
\hline 13 & 0 & 0 & 0 & 0 & 0 & 0 & 0 & 0 & 0.00128 & 0.00509 & 0.02741 & 0.40590 & 0.21283 \\
\hline 14 & 0 & 0 & 0 & 0 & 0 & 0 & 0 & 0.00001 & 0.00004 & 0.00136 & 0.00931 & 0.01604 & 0.39937 \\
\hline 15 & 0 & 0 & 0 & 0 & 0 & 0 & 0 & 0 & 0.00002 & 0.00012 & 0.00120 & 0.01480 & 0.00609 \\
\hline 16 & 0 & 0 & 0 & 0 & 0 & 0 & 0 & 0 & 0 & 0.00002 & 0.00029 & 0.00078 & 0.02098 \\
\hline 17 & 0 & 0 & 0 & 0 & 0 & 0 & 0 & 0 & 0 & 0 & 0.00001 & 0.00056 & 0.00026 \\
\hline 18 & 0 & 0 & 0 & 0 & 0 & 0 & 0 & 0 & 0 & 0 & 0.00001 & 0 & 0.00089 \\
\hline 19 & 0 & 0 & 0 & 0 & 0 & 0 & 0 & 0 & 0 & 0 & 0 & 0.00002 & 0 \\
\hline 20 & 0 & 0 & 0 & 0 & 0 & 0 & 0 & 0 & 0 & 0 & 0 & 0 & 0.00002 \\
\hline 21 & 0 & 0 & 0 & 0 & 0 & 0 & 0 & 0 & 0 & 0 & 0 & 0 & 0 \\
\hline 22 & 0 & 0 & 0 & 0 & 0 & 0 & 0 & 0 & 0 & 0 & 0 & 0 & 0 \\
\hline 23 & 0 & 0 & 0 & 0 & 0 & 0 & 0 & 0 & 0 & 0 & 0 & 0 & 0 \\
\hline 24 & 0 & 0 & 0 & 0 & 0 & 0 & 0 & 0 & 0 & 0 & 0 & 0 & 0 \\
\hline
\end{tabular}


Table A.4: Franck-Condon factors for the B $-\mathrm{X}$ transition, part 2 (from $v^{\prime \prime}=13$ to $v^{\prime \prime}=24$ ).

\begin{tabular}{|c|c|c|c|c|c|c|c|c|c|c|c|c|}
\hline$v^{\prime}$ & $v^{\prime \prime}=13$ & $v^{\prime \prime}=14$ & $v^{\prime \prime}=15$ & $v^{\prime \prime}=16$ & $v^{\prime \prime}=17$ & $v^{\prime \prime}=18$ & $v^{\prime \prime}=19$ & $v^{\prime \prime}=20$ & $v^{\prime \prime}=21$ & $v^{\prime \prime}=22$ & $v^{\prime \prime}=23$ & $v^{\prime \prime}=24$ \\
\hline 0 & 0 & 0 & 0 & 0 & 0 & 0 & 0 & 0 & 0 & 0 & 0 & 0 \\
\hline 1 & 0 & 0 & 0 & 0 & 0 & 0 & 0 & 0 & 0 & 0 & 0 & 0 \\
\hline 2 & 0 & 0 & 0 & 0 & 0 & 0 & 0 & 0 & 0 & 0 & 0 & 0 \\
\hline 3 & 0 & 0 & 0 & 0 & 0 & 0 & 0 & 0 & 0 & 0 & 0 & 0 \\
\hline 4 & 0 & 0 & 0 & 0 & 0 & 0 & 0 & 0 & 0 & 0 & 0 & 0 \\
\hline 5 & 0.00016 & 0.00001 & 0 & 0 & 0 & 0 & 0 & 0 & 0 & 0 & 0 & 0 \\
\hline 6 & 0.00171 & 0.00026 & 0.00002 & 0 & 0 & 0 & 0 & 0 & 0 & 0 & 0 & 0 \\
\hline 7 & 0.01024 & 0.00238 & 0.00038 & 0.00004 & 0 & 0 & 0 & 0 & 0 & 0 & 0 & 0 \\
\hline 8 & 0.03847 & 0.01258 & 0.00308 & 0.00052 & 0.00005 & 0 & 0 & 0 & 0 & 0 & 0 & 0 \\
\hline 9 & 0.08830 & 0.04264 & 0.01472 & 0.00382 & 0.00067 & 0.00007 & 0 & 0 & 0 & 0 & 0 & 0 \\
\hline 10 & 0.11158 & 0.08980 & 0.04587 & 0.01667 & 0.00451 & 0.00083 & 0.00009 & 0 & 0 & 0 & 0 & 0 \\
\hline 11 & 0.05294 & 0.10520 & 0.09004 & 0.04824 & 0.01829 & 0.00513 & 0.00097 & 0.00011 & 0 & 0 & 0 & 0 \\
\hline 12 & 0.00217 & 0.04728 & 0.09918 & 0.08943 & 0.04976 & 0.01958 & 0.00565 & 0.00110 & 0.00012 & 0 & 0 & 0 \\
\hline 13 & 0.04003 & 0.00335 & 0.04384 & 0.09370 & 0.08817 & 0.05052 & 0.02048 & 0.00605 & 0.00119 & 0.00013 & 0 & 0.00001 \\
\hline 14 & 0.24413 & 0.02827 & 0.00453 & 0.04238 & 0.08873 & 0.08650 & 0.05061 & 0.02098 & 0.00631 & 0.00125 & 0.00013 & 0 \\
\hline 15 & 0.38179 & 0.28246 & 0.01706 & 0.00577 & 0.04286 & 0.08412 & 0.08467 & 0.05002 & 0.02116 & 0.00640 & 0.00129 & 0.00012 \\
\hline 16 & 0.00041 & 0.35107 & 0.32611 & 0.00764 & 0.00722 & 0.04532 & 0.07973 & 0.08273 & 0.04890 & 0.02095 & 0.00639 & 0.00126 \\
\hline 17 & 0.02673 & 0.00209 & 0.30626 & 0.37203 & 0.00149 & 0.00908 & 0.05005 & 0.07523 & 0.08090 & 0.04721 & 0.02046 & 0.00620 \\
\hline 18 & 0 & 0.03057 & 0.01384 & 0.24832 & 0.41548 & 0.00023 & 0.01161 & 0.05749 & 0.07033 & 0.07930 & 0.04500 & 0.01972 \\
\hline 19 & 0.00119 & 0.00056 & 0.03090 & 0.03695 & 0.18115 & 0.45010 & 0.00526 & 0.01508 & 0.06830 & 0.06467 & 0.07809 & 0.04227 \\
\hline 20 & 0.00005 & 0.00132 & 0.00255 & 0.02673 & 0.07029 & 0.11200 & 0.46840 & 0.01735 & 0.01976 & 0.08332 & 0.05796 & 0.07752 \\
\hline 21 & 0.00002 & 0.00018 & 0.00114 & 0.00634 & 0.01839 & 0.10937 & 0.05119 & 0.46317 & 0.03604 & 0.02579 & 0.10353 & 0.04996 \\
\hline 22 & 0 & 0.00002 & 0.00043 & 0.00064 & 0.01166 & 0.00828 & 0.14629 & 0.01063 & 0.42935 & 0.05911 & 0.03296 & 0.12986 \\
\hline 23 & 0 & 0.00001 & 0 & 0.00076 & 0.00011 & 0.01727 & 0.00091 & 0.17093 & 0.00089 & 0.36627 & 0.08246 & 0.04054 \\
\hline 24 & 0 & 0 & 0.00002 & 0.00001 & 0.00105 & 0.00014 & 0.02110 & 0.00197 & 0.17375 & 0.02719 & 0.27989 & 0.10055 \\
\hline
\end{tabular}


Table A.5: Transition probabilities $A_{i k}^{v^{\prime} v^{\prime \prime}}\left(\mathrm{s}^{-1}\right)$ for the $\mathrm{A}-\mathrm{X}$ transition, part 1 (from $v^{\prime \prime}=0$ to $v^{\prime \prime}=12$ ).

\begin{tabular}{|c|c|c|c|c|c|c|c|c|c|c|c|c|c|}
\hline$v^{\prime}$ & $v^{\prime \prime}=0$ & $v^{\prime \prime}=1$ & $v^{\prime \prime}=2$ & $v^{\prime \prime}=3$ & $v^{\prime \prime}=4$ & $v^{\prime \prime}=5$ & $v^{\prime \prime}=6$ & $v^{\prime \prime}=7$ & $v^{\prime \prime}=8$ & $v^{\prime \prime}=9$ & $v^{\prime \prime}=10$ & $v^{\prime \prime}=11$ & $v^{\prime \prime}=12$ \\
\hline 0 & 90202 & 40403 & 11117 & 2373 & 414 & 64 & 8 & 0 & 0 & 0 & 0 & 0 & 0 \\
\hline 1 & 74083 & 16386 & 36689 & 18893 & 5901 & 1367 & 260 & 40 & 5 & 0 & 0 & 0 & 0 \\
\hline 2 & 23201 & 82794 & 167 & 22938 & 21111 & 9191 & 2702 & 621 & 111 & 16 & 2 & 0 & 0 \\
\hline 3 & 3332 & 49079 & 68670 & 3778 & 11047 & 19349 & 11438 & 4169 & 1128 & 234 & 40 & 5 & 0 \\
\hline 4 & 199 & 10034 & 70451 & 50020 & 11527 & 3769 & 15620 & 12476 & 5516 & 1744 & 410 & 78 & 11 \\
\hline 5 & 3 & 730 & 19140 & 85981 & 33722 & 17817 & 550 & 11462 & 12419 & 6615 & 2393 & 637 & 135 \\
\hline 6 & 0 & 9 & 1604 & 29602 & 96538 & 21552 & 21352 & 61 & 7716 & 11601 & 7365 & 3028 & 900 \\
\hline 7 & 0 & 0 & 15 & 2740 & 40583 & 103580 & 13272 & 22438 & 1065 & 4752 & 10337 & 7758 & 3607 \\
\hline 8 & 0 & 0 & 2 & 18 & 3997 & 51500 & 108570 & 8014 & 21792 & 2691 & 2640 & 8858 & 7867 \\
\hline 9 & 0 & 0 & 0 & 6 & 13 & 5208 & 61982 & 112660 & 4871 & 20111 & 4400 & 1264 & 7386 \\
\hline 10 & 0 & 0 & 0 & 0 & 14 & 3 & 6205 & 71771 & 116760 & 3114 & 17909 & 5923 & 473 \\
\hline 11 & 0 & 0 & 0 & 0 & 0 & 30 & 2 & 6840 & 80660 & 121540 & 2232 & 15523 & 7157 \\
\hline 12 & 0 & 0 & 0 & 0 & 0 & 0 & 54 & 34 & 6997 & 88441 & 127450 & 1912 & 13161 \\
\hline 13 & 0 & 0 & 0 & 0 & 0 & 0 & 0 & 88 & 140 & 6612 & 94888 & 134830 & 2024 \\
\hline 14 & 0 & 0 & 0 & 0 & 0 & 0 & 0 & 0 & 127 & 368 & 5692 & 99703 & 143850 \\
\hline 15 & 0 & 0 & 0 & 0 & 0 & 0 & 0 & 0 & 0 & 166 & 772 & 4333 & 102510 \\
\hline 16 & 0 & 0 & 0 & 0 & 0 & 0 & 0 & 0 & 1 & 3 & 193 & 1388 & 2733 \\
\hline 17 & 0 & 0 & 0 & 0 & 0 & 0 & 0 & 0 & 0 & 2 & 10 & 194 & 2231 \\
\hline 18 & 0 & 0 & 0 & 0 & 0 & 0 & 0 & 0 & 0 & 0 & 2 & 26 & 163 \\
\hline 19 & 0 & 0 & 0 & 0 & 0 & 0 & 0 & 0 & 0 & 0 & 0 & 3 & 54 \\
\hline 20 & 0 & 0 & 0 & 0 & 0 & 0 & 0 & 0 & 0 & 0 & 0 & 0 & 2 \\
\hline 21 & 0 & 0 & 0 & 0 & 0 & 0 & 0 & 0 & 0 & 0 & 0 & 0 & 2 \\
\hline 22 & 0 & 0 & 0 & 0 & 0 & 0 & 0 & 0 & 0 & 0 & 0 & 0 & 0 \\
\hline 23 & 0 & 0 & 0 & 0 & 0 & 0 & 0 & 0 & 0 & 0 & 0 & 0 & 0 \\
\hline 24 & 0 & 0 & 0 & 0 & 0 & 0 & 0 & 0 & 0 & 0 & 0 & 0 & 0 \\
\hline
\end{tabular}


Table A.6: Transition probabilities $A_{i k}^{v^{\prime} v^{\prime \prime}}\left(\mathrm{s}^{-1}\right)$ for the A $-\mathrm{X}$ transition, part 2 (from $v^{\prime \prime}=13$ to $v^{\prime \prime}=24$ ).

\begin{tabular}{|c|c|c|c|c|c|c|c|c|c|c|c|c|}
\hline$v^{\prime}$ & $v^{\prime \prime}=13$ & $v^{\prime \prime}=14$ & $v^{\prime \prime}=15$ & $v^{\prime \prime}=16$ & $v^{\prime \prime}=17$ & $v^{\prime \prime}=18$ & $v^{\prime \prime}=19$ & $v^{\prime \prime}=20$ & $v^{\prime \prime}=21$ & $v^{\prime \prime}=22$ & $v^{\prime \prime}=23$ & $v^{\prime \prime}=24$ \\
\hline 0 & 0 & 0 & 0 & 0 & 0 & 0 & 0 & 0 & 0 & 0 & 0 & 0 \\
\hline 1 & 0 & 0 & 0 & 0 & 0 & 0 & 0 & 0 & 0 & 0 & 0 & 0 \\
\hline 2 & 0 & 0 & 0 & 0 & 0 & 0 & 0 & 0 & 0 & 0 & 0 & 0 \\
\hline 3 & 0 & 0 & 0 & 0 & 0 & 0 & 0 & 0 & 0 & 0 & 0 & 0 \\
\hline 4 & 0 & 0 & 0 & 0 & 0 & 0 & 0 & 0 & 0 & 0 & 0 & 0 \\
\hline 5 & 21 & 2 & 0 & 0 & 0 & 0 & 0 & 0 & 0 & 0 & 0 & 0 \\
\hline 6 & 209 & 36 & 4 & 0 & 0 & 0 & 0 & 0 & 0 & 0 & 0 & 0 \\
\hline 7 & 1179 & 301 & 56 & 7 & 0 & 0 & 0 & 0 & 0 & 0 & 0 & 0 \\
\hline 8 & 4077 & 1472 & 404 & 81 & 11 & 0 & 0 & 0 & 0 & 0 & 0 & 0 \\
\hline 9 & 7711 & 4455 & 1744 & 519 & 111 & 17 & 1 & 0 & 0 & 0 & 0 & 0 \\
\hline 10 & 6016 & 7385 & 4715 & 1997 & 638 & 145 & 24 & 2 & 0 & 0 & 0 & 0 \\
\hline 11 & 101 & 4807 & 6958 & 4858 & 2227 & 752 & 184 & 32 & 3 & 0 & 0 & 0 \\
\hline 12 & 8088 & 0 & 3800 & 6449 & 4933 & 2404 & 869 & 222 & 42 & 4 & 0 & 0 \\
\hline 13 & 10906 & 8772 & 58 & 2967 & 5945 & 4904 & 2565 & 965 & 265 & 51 & 5 & 0 \\
\hline 14 & 2582 & 8807 & 9266 & 192 & 2311 & 5433 & 4837 & 2668 & 1061 & 302 & 62 & 6 \\
\hline 15 & 154570 & 3723 & 6864 & 9654 & 340 & 1797 & 4956 & 4721 & 2740 & 1139 & 338 & 73 \\
\hline 16 & 102910 & 166870 & 5697 & 5074 & 10013 & 464 & 1399 & 4525 & 4572 & 2782 & 1201 & 371 \\
\hline 17 & 1207 & 100480 & 180450 & 8843 & 3447 & 10419 & 542 & 1097 & 4138 & 4411 & 2786 & 1255 \\
\hline 18 & 3265 & 176 & 94895 & 194750 & 13579 & 2016 & 10957 & 557 & 864 & 3810 & 4230 & 2775 \\
\hline 19 & 101 & 4396 & 142 & 85970 & 208880 & 20355 & 866 & 11717 & 507 & 683 & 3536 & 4042 \\
\hline 20 & 96 & 32 & 5455 & 1623 & 73854 & 221670 & 29591 & 142 & 12802 & 400 & 538 & 3313 \\
\hline 21 & 0 & 147 & 0 & 6224 & 5045 & 59104 & 231550 & 41580 & 63 & 14345 & 252 & 415 \\
\hline 22 & 3 & 0 & 200 & 83 & 6468 & 10616 & 42818 & 236830 & 56372 & 927 & 16511 & 101 \\
\hline 23 & 0 & 5 & 5 & 238 & 368 & 6020 & 18186 & 26643 & 235780 & 73608 & 3092 & 19503 \\
\hline 24 & 0 & 0 & 5 & 20 & 244 & 930 & 4850 & 27148 & 12670 & 226990 & 92393 & 6924 \\
\hline
\end{tabular}


Table A.7: Transition probabilities $A_{i k}^{v^{\prime} v^{\prime \prime}}\left(\mathrm{s}^{-1}\right)$ for the $\mathrm{B}-\mathrm{X}$ transition, part 1 (from $v^{\prime \prime}=0$ to $v^{\prime \prime}=12$ ).

\begin{tabular}{|c|c|c|c|c|c|c|c|c|c|c|c|c|c|}
\hline$v^{\prime}$ & $v^{\prime \prime}=0$ & $v^{\prime \prime}=1$ & $v^{\prime \prime}=2$ & $v^{\prime \prime}=3$ & $v^{\prime \prime}=4$ & $v^{\prime \prime}=5$ & $v^{\prime \prime}=6$ & $v^{\prime \prime}=7$ & $v^{\prime \prime}=8$ & $v^{\prime \prime}=9$ & $v^{\prime \prime}=10$ & $v^{\prime \prime}=11$ & $v^{\prime \prime}=12$ \\
\hline 0 & 134930 & 62014 & 17766 & 3949 & 719 & 115 & 15 & 2 & 0 & 0 & 0 & 0 & 0 \\
\hline 1 & 75882 & 36375 & 63314 & 31917 & 10070 & 2384 & 460 & 71 & 9 & 0 & 0 & 0 & 0 \\
\hline 2 & 15180 & 92910 & 4983 & 47479 & 38512 & 16233 & 4770 & 1084 & 196 & 28 & 3 & 0 & 0 \\
\hline 3 & 1150 & 32663 & 87339 & 98 & 30759 & 39073 & 21195 & 7465 & 1974 & 401 & 65 & 8 & 0 \\
\hline 4 & 18 & 3272 & 47984 & 75186 & 3921 & 17933 & 36096 & 24495 & 10135 & 3042 & 692 & 124 & 16 \\
\hline 5 & 0 & 48 & 5863 & 60211 & 62985 & 9223 & 9451 & 31533 & 26246 & 12500 & 4206 & 1053 & 206 \\
\hline 6 & 0 & 2 & 69 & 8434 & 69684 & 53023 & 13451 & 4401 & 26682 & 26730 & 14454 & 5353 & 1471 \\
\hline 7 & 0 & 0 & 10 & 65 & 10608 & 77061 & 45802 & 16015 & 1701 & 22194 & 26361 & 15912 & 6444 \\
\hline 8 & 0 & 0 & 0 & 28 & 33 & 12119 & 82959 & 41187 & 17029 & 455 & 18385 & 25431 & 16935 \\
\hline 9 & 0 & 0 & 0 & 1 & 63 & 2 & 12797 & 87796 & 38892 & 16807 & 35 & 15312 & 24226 \\
\hline 10 & 0 & 0 & 0 & 0 & 1 & 119 & 27 & 12549 & 91751 & 38678 & 15658 & 36 & 12952 \\
\hline 11 & 0 & 0 & 0 & 0 & 0 & 0 & 192 & 189 & 11371 & 94798 & 40392 & 13844 & 223 \\
\hline 12 & 0 & 0 & 0 & 0 & 0 & 0 & 0 & 271 & 583 & 9363 & 96707 & 43968 & 11568 \\
\hline 13 & 0 & 0 & 0 & 0 & 0 & 0 & 2 & 1 & 335 & 1293 & 6757 & 97078 & 49379 \\
\hline 14 & 0 & 0 & 0 & 0 & 0 & 0 & 0 & 4 & 10 & 356 & 2361 & 3947 & 95365 \\
\hline 15 & 0 & 0 & 0 & 0 & 0 & 0 & 0 & 0 & 5 & 33 & 314 & 3745 & 1495 \\
\hline 16 & 0 & 0 & 0 & 0 & 0 & 0 & 0 & 0 & 0 & 5 & 78 & 204 & 5295 \\
\hline 17 & 0 & 0 & 0 & 0 & 0 & 0 & 0 & 0 & 0 & 1 & 3 & 149 & 68 \\
\hline 18 & 0 & 0 & 0 & 0 & 0 & 0 & 0 & 0 & 0 & 0 & 3 & 0 & 237 \\
\hline 19 & 0 & 0 & 0 & 0 & 0 & 0 & 0 & 0 & 0 & 0 & 0 & 5 & 1 \\
\hline 20 & 0 & 0 & 0 & 0 & 0 & 0 & 0 & 0 & 0 & 0 & 0 & 0 & 7 \\
\hline 21 & 0 & 0 & 0 & 0 & 0 & 0 & 0 & 0 & 0 & 0 & 0 & 0 & 0 \\
\hline 22 & 0 & 0 & 0 & 0 & 0 & 0 & 0 & 0 & 0 & 0 & 0 & 0 & 0 \\
\hline 23 & 0 & 0 & 0 & 0 & 0 & 0 & 0 & 0 & 0 & 0 & 0 & 0 & 0 \\
\hline 24 & 0 & 0 & 0 & 0 & 0 & 0 & 0 & 0 & 0 & 0 & 0 & 0 & 0 \\
\hline
\end{tabular}


Table A.8: Transition probabilities $A_{i k}^{v^{\prime} v^{\prime \prime}}\left(\mathrm{s}^{-1}\right)$ for the $\mathrm{B}-\mathrm{X}$ transition, part 2 (from $v^{\prime \prime}=13$ to $v^{\prime \prime}=24$ ).

\begin{tabular}{|c|c|c|c|c|c|c|c|c|c|c|c|c|}
\hline$v^{\prime}$ & $v^{\prime \prime}=13$ & $v^{\prime \prime}=14$ & $v^{\prime \prime}=15$ & $v^{\prime \prime}=16$ & $v^{\prime \prime}=17$ & $v^{\prime \prime}=18$ & $v^{\prime \prime}=19$ & $v^{\prime \prime}=20$ & $v^{\prime \prime}=21$ & $v^{\prime \prime}=22$ & $v^{\prime \prime}=23$ & $v^{\prime \prime}=24$ \\
\hline 0 & 0 & 0 & 0 & 0 & 0 & 0 & 0 & 0 & 0 & 0 & 0 & 0 \\
\hline 1 & 0 & 0 & 0 & 0 & 0 & 0 & 0 & 0 & 0 & 0 & 0 & 0 \\
\hline 2 & 0 & 0 & 0 & 0 & 0 & 0 & 0 & 0 & 0 & 0 & 0 & 0 \\
\hline 3 & 0 & 0 & 0 & 0 & 0 & 0 & 0 & 0 & 0 & 0 & 0 & 0 \\
\hline 4 & 1 & 0 & 0 & 0 & 0 & 0 & 0 & 0 & 0 & 0 & 0 & 0 \\
\hline 5 & 28 & 2 & 0 & 0 & 0 & 0 & 0 & 0 & 0 & 0 & 0 & 0 \\
\hline 6 & 307 & 45 & 4 & 0 & 0 & 0 & 0 & 0 & 0 & 0 & 0 & 0 \\
\hline 7 & 1904 & 428 & 66 & 6 & 0 & 0 & 0 & 0 & 0 & 0 & 0 & 0 \\
\hline 8 & 7398 & 2342 & 555 & 91 & 9 & 0 & 0 & 0 & 0 & 0 & 0 & 0 \\
\hline 9 & 17550 & 8209 & 2745 & 689 & 118 & 12 & 0 & 0 & 0 & 0 & 0 & 0 \\
\hline 10 & 22904 & 17860 & 8839 & 3112 & 815 & 145 & 15 & 0 & 0 & 0 & 0 & 0 \\
\hline 11 & 11213 & 21599 & 17918 & 9303 & 3418 & 929 & 170 & 18 & 0 & 0 & 0 & 0 \\
\hline 12 & 473 & 10014 & 20364 & 17800 & 9600 & 3661 & 1024 & 193 & 20 & 0 & 1 & 1 \\
\hline 13 & 9008 & 732 & 9279 & 19233 & 17548 & 9748 & 3831 & 1096 & 210 & 22 & 0 & 2 \\
\hline 14 & 56563 & 6354 & 988 & 8965 & 18204 & 17211 & 9764 & 3926 & 1145 & 220 & 22 & 0 \\
\hline 15 & 91003 & 65337 & 3828 & 1257 & 9057 & 17245 & 16837 & 9647 & 3959 & 1162 & 226 & 21 \\
\hline 16 & 100 & 83509 & 75293 & 1711 & 1570 & 9565 & 16328 & 16439 & 9427 & 3918 & 1159 & 222 \\
\hline 17 & 6729 & 512 & 72681 & 85714 & 334 & 1972 & 10546 & 15387 & 16057 & 9093 & 3825 & 1125 \\
\hline 18 & 0 & 7673 & 3373 & 58780 & 95500 & 51 & 2516 & 12093 & 14361 & 15718 & 8658 & 3683 \\
\hline 19 & 317 & 144 & 7731 & 8981 & 42761 & 103190 & 1172 & 3261 & 14338 & 13182 & 15455 & 8122 \\
\hline 20 & 13 & 348 & 655 & 6665 & 17028 & 26357 & 107080 & 3853 & 4262 & 17452 & 11790 & 15313 \\
\hline 21 & 7 & 50 & 299 & 1620 & 4569 & 26403 & 12008 & 105550 & 7979 & 5546 & 21631 & 10138 \\
\hline 22 & 2 & 4 & 116 & 168 & 2968 & 2049 & 35185 & 2485 & 97518 & 13046 & 7069 & 27057 \\
\hline 23 & 0 & 4 & 0 & 205 & 28 & 4380 & 223 & 40948 & 206 & 82892 & 18137 & 8667 \\
\hline 24 & 0 & 0 & 6 & 3 & 282 & 35 & 5326 & 483 & 41447 & 6306 & 63097 & 22034 \\
\hline
\end{tabular}

